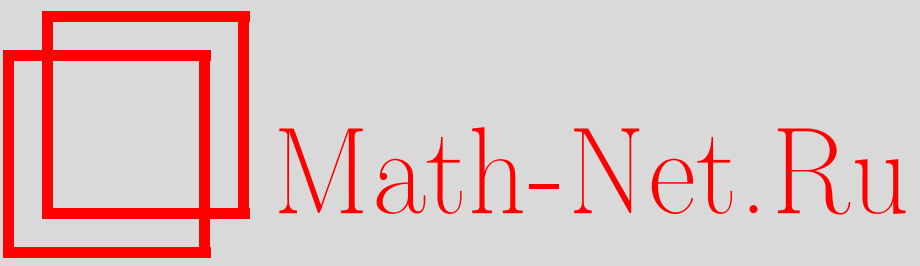

А. В. Никифорова, Контактно-проективные преобразования почти контактной метрической структуры, Итоги науки и техн. Сер. Соврем. мат. и ее прил. Темат. обз., 2020, том 180, 66-68

DOI: https://doi.org/10.36535/0233-6723-2020-180-66-68

Использование Общероссийского математического портала Math-Net.Ru подразумевает, что вы прочитали и согласны с пользовательским соглашением

http://www.mathnet.ru/rus/agreement

Параметры загрузки:

IP : 54.157 .27 .8

26 апреля 2023 г., 13:22:12 


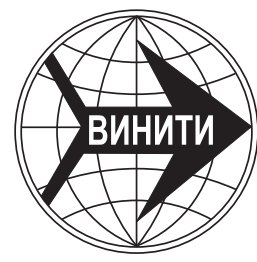

ИТОГИ НАУКИ И ТЕХНИКИ.

Современная математика и ее приложения.

Тематические обзоры.

Том 180 (2020). С. 66-68

DOI: $10.36535 / 0233-6723-2020-180-66-68$

УДК 514.76

\title{
КОНТАКТНО-ПРОЕКТИВНЫЕ ПРЕОБРАЗОВАНИЯ ПОЧТИ КОНТАКТНОЙ МЕТРИЧЕСКОЙ СТРУКТУРЫ
}

\author{
(c) 2020 г. $\quad$ А. В. НИКИФОРОВА
}

\begin{abstract}
АннотАция. В статье рассматривается вопрос о формулах перехода от одной почти контактной метрической структуры к другой. Определены условия, которые накладываются на четверку тензорных полей почти контактной метрической структуры.
\end{abstract}

Ключевые слова: контактно-проективное преобразование, почти контактная метрическая структура.

\section{CONTACT-PROJECTIVE TRANSFORMATIONS OF ALMOST CONTACT METRIC STRUCTURE}

\author{
(c) 2020 A. V. NIKIFOROVA
}

\begin{abstract}
In this paper, the problem on the transition from one almost contact metric structure to another in examined. We obtain conditions for four tensor fields of an almost contact metric structure.
\end{abstract}

Keywords and phrases: contact-projective transformation, almost contact metric structure.

AMS Subject Classification: 53B05, 51E15

Пусть $M$ - почти эрмитово многообразие, т.е. связное гладкое $2 n$-мерное многообразие, на котором определена почти эрмитова структура - пара тензорных полей $(J, g)$, где $J$ - тензорное поле типа $(1,1)$, обладающее свойством $J^{2}=-\mathrm{id}$, а $g$-риманова метрика.

Голоморфно-проективным преобразованием почти эрмитова многообразия называется такой переход от почти эрмитовой структуры $(J, g)$ к почти эрмитовой структуре $(J, \tilde{g})$, при котором обе структуры имеют общие голоморфно почти геодезические кривые, т.е. такие кривые многообразия $M$, для которых любой касательный вектор $\mu_{p}$ после параллельного переноса из точки $p$ в произвольную точку $q$ на $M$ принадлежит линейной оболочке $L\left(\mu_{q}, J \mu_{q}\right)$ (см. [1]).

Известно, что на произвольной гиперповерхности $N$ почти эрмитова многообразия $M$ естественным образом возникает почти контактная метрическая структура ( $A C$-структура; см. [2]), т.е. такая четверка тензорных полей $(\Phi, \xi, \eta, g)$, где $\Phi$ - тензорное поле типа $(1,1), \eta$ - 1 -форма, $\xi$ - векторное поле, $g$ - риманова метрика, что выполняются соотношения

$$
\Phi(\xi)=0, \quad \eta \circ \Phi=0, \quad \eta(\xi)=1, \quad \Phi^{2}=-\mathrm{id}+\xi \otimes \eta, \quad g(\Phi X, \Phi Y)=g(X, Y)-\eta(X) \eta(Y) .
$$

Таким образом, гладкое многообразие $N$ становится почти контактным метрическим многообразием.

Голоморфно-проективное преобразование почти эрмитовой структуры $(J, g)$ произвольного многообразия $M$ индуцирует на гиперповерхности $N$ преобразование $A C$-структуры $(g, \Phi, \xi, \eta)$, возникающей на $N$ следующим образом. Пусть $\nu$ - поле единичной нормали; положим

$$
\xi=J(\nu), \quad \eta(X)=g(\xi, X), \quad \zeta(X)=g(\nu, X), \quad \Phi=\left.J \circ(\mathrm{id}-\xi \otimes \nu-\eta \otimes \xi)\right|_{\mathfrak{X}(M)} .
$$


В этом случае связь между старой $A C$-структурой $(g, \Phi, \xi, \eta)$ и новой $A C$-структурой $(\tilde{g}, \tilde{\Phi}, \tilde{\xi}, \tilde{\eta})$ многообразия $N$ выражается следующими соотношениями (см. [3]):

$$
\tilde{g}(X, Y)=g(X, f Y), \quad \tilde{\xi}=\lambda f^{-1}(\xi), \quad \tilde{\eta}=\lambda \eta, \quad \tilde{\Phi}=\Phi-\eta \otimes \nu+\lambda^{2} \eta \otimes f^{-1} \nu,
$$

где $f$ - тензор голоморфно-проективной деформации,

$$
\lambda= \pm \sqrt{\left(\zeta\left(f^{-1} \nu\right)\right)^{-1}}
$$

Такой переход называется контактно-проективным преобразованием (см. [3]).

Рассмотрим теперь произвольную $A C$-структуру $(g, \Phi, \xi, \eta)$ и выясним, когда формулы $(2)$, где $f$ - произвольный невырожденный эндоморфизм, $\nu$ - векторное поле, $\lambda$ - гладкая функция, определяют переход к новой $A C$-структуре $(\tilde{g}, \tilde{\Phi}, \tilde{\xi}, \tilde{\eta})$. Подставляя в первые три равенства, определенные формулами (1), соответствующие выражения из формул (2), после преобразований получим

$$
\eta\left(f^{-1}(\xi)\right)=\frac{1}{\lambda^{2}}, \quad \Phi\left(f^{-1} \xi\right)=\frac{1}{\lambda^{2}} \vartheta,
$$

где введено обозначение $\vartheta=\nu-\lambda^{2} f^{-1} \nu$;

$$
\xi-\lambda^{2} f^{-1} \xi=\Phi \vartheta
$$

$\lambda$ не обращается в нуль ни в какой точке многообразия.

Заметим, что четвертое равенство в условии (2) с учетом введенного обозначения можно записать в виде

$$
\tilde{\Phi}=\Phi-\eta \otimes \vartheta
$$

Подставим это выражение и выражение $\tilde{\eta}$ из формул (2) в равенство $\tilde{\eta} \circ \tilde{\Phi}=0$ :

$$
(\lambda \eta) \circ(\Phi-\eta \otimes \vartheta)=0 .
$$

Тогда для произвольного векторного поля $X$ с учетом $\eta \circ \Phi=0$ получим $\lambda \eta(X) \eta(\vartheta)=0$. Положим $X=\xi$. Так как $\eta(\xi)=1$, то имеем условие $\eta(\vartheta)=0$, откуда следует, что $\vartheta \in L$, где $L-$ первое фундаментальное распределение почти контактной метрической структуры.

Далее, подставляя в равенство

$$
\tilde{g}(\tilde{\Phi} X, \tilde{\Phi} Y)=\tilde{g}(X, Y)-\tilde{\nu}(X) \tilde{\nu}(Y)
$$

определенные выше выражения для $\tilde{g}, \tilde{\eta}$ и $\tilde{\Phi}$, получим

$$
g(\Phi X, f \Phi)-\eta(X) g(\vartheta, f \Phi Y)-\eta(Y) g(\Phi X, f \vartheta)+\eta(X) \eta(Y) g(\vartheta, f \vartheta)=g(X, f Y)-\lambda^{2} \nu(X) \nu(Y) .
$$

Тогда имеем

Подставим теперь в равенство

$$
g(\Phi \vartheta, f \Phi \vartheta)=g(\vartheta, f \vartheta)
$$

$$
\tilde{\Phi}^{2}=-\mathrm{id}+\tilde{\eta} \otimes \tilde{\xi}
$$

выражения $\tilde{\eta}, \tilde{\xi}$ и $\tilde{\Phi}$, определенные выше:

$$
(\Phi-\eta \otimes \vartheta)^{2}=-\mathrm{id}+\lambda \eta \otimes \lambda f^{-1} \xi .
$$

Учитывая, что $\Phi^{2}=-\mathrm{id}+\eta \otimes \xi$ и $\eta \circ \Phi=0$, получим

$$
\eta \otimes \xi-\lambda^{2} \eta \otimes f^{-1} \xi-\eta \otimes \Phi \vartheta-\eta(\vartheta)(\eta \otimes \vartheta)=0 .
$$

Отсюда следует, что

$$
\eta \otimes\left(\xi-\lambda^{2} f^{-1} \xi\right)-\eta \otimes \Phi \vartheta-\eta(\vartheta)(\eta \otimes \vartheta)=0 .
$$

Так как имеют место формулы $\xi-\lambda^{2} f^{-1} \xi=\Phi \vartheta$ и $\eta(\vartheta)=0$, то полученное условие выполняется тождественно; следовательно, условие

$$
\tilde{\Phi}^{2}=-\mathrm{id}+\tilde{\eta} \otimes \tilde{\xi}
$$

выполняется тождественно. 
Теорема 1. Формули (2) определяют переход от $A C$-структури $(g, \Phi, \xi, \eta) \propto$ новой $A C$ структуре $(\tilde{g}, \tilde{\Phi}, \tilde{\xi}, \tilde{\eta})$ тогда и только тогда, когда выполняются условия

$$
\eta\left(f^{-1}(\xi)\right)=\frac{1}{\lambda^{2}}, \quad \Phi\left(f^{-1} \xi\right)=\frac{1}{\lambda^{2}} \vartheta, \quad \eta(\vartheta)=0, \quad g(\Phi \vartheta, f \Phi \vartheta)=g(\vartheta, f \vartheta),
$$

где $\vartheta=\nu-\lambda^{2} f^{-1} \nu$.

Назовем переход от одной почти контактной метрической структуры к другой при выполнении полученных условий обобщенным контактно-проективным преобразованием $A C$-структуры. Примером такого преобразования может также служить конформное преобразование почти контактной метрической структуры, т.е. переход от почти контактной метрической структуры $(\Phi, \xi, \eta, g)$ к почти контактной метрической структуре $\left(\Phi, \tilde{\xi}=e^{-f} \xi, \tilde{\eta}=e^{f} \eta, \tilde{g}=e^{2 f} g\right)$, где $f-$ гладкая функция на многообразии $M$. Для него полученные условия выполняются.

Кроме того, имеют место следующие утверждения.

Теорема 2. Если $f$-скалярный эндоморфизм с собственным значением $\mu$, заданный на почти контактном метрическом многообразии, то

(1) $\mu>0$, причем $\mu=\lambda^{2}$;

(2) $f$-конформное преобразование AC-структуры.

Теорема 3. Пусть $\xi$-собственное векторное поле эндоморфизма $f$ с собственным значением $\beta$. Тогда

(1) $\beta=\lambda^{2}$;

(2) $\nu$-собственный вектор эндоморфизма с тем же собственным значением $\lambda^{2}$;

(3) формуль преобразования AC-структуры имеют вид

$$
\tilde{g}(X, Y)=g(X, f Y), \quad \tilde{\xi}=\frac{1}{\lambda} \xi, \quad \tilde{\eta}=\lambda \eta, \quad \tilde{\Phi}=\Phi .
$$

\section{СПИСОК ЛИТЕРАТУРЫ}

1. Синюков Н. С. Геодезические отображения римановых пространств. - М.: Наука, 1979.

2. Кириченко В. Ф. Дифференциально-геометрические структуры на многообразиях. - Одесса: Печатный дом, 2013.

3. Nikiforova A. V., Ignatochkina L. A. The transformations induced on hypersurfaces of almost Hermitian manifolds// J. Basrah Res. - 2011. - 37, № 4C. - P. 1--7.

Никифорова Анна Валентиновна

Московский педагогический государственный университет

E-mail: anik7@bk.ru 